\title{
Colloidal electrostatic interactions near a conducting surface
}

\author{
Marco Polin, ${ }^{1}$ David G. Grier, ${ }^{1}$ and Yilong $\operatorname{Han}^{2}$ \\ ${ }^{1}$ Center for Soft Matter Research, New York University, 4 Washington Place, New York, New York 10003, USA \\ ${ }^{2}$ Department of Physics, Hong Kong University of Science and Technology, Clear Water Bay, Kowloon, Hong Kong
}

(Received 2 May 2006; revised manuscript received 19 July 2007; published 30 October 2007)

\begin{abstract}
Like-charged colloidal spheres dispersed in de-ionized water are supposed to repel each other. Instead, artifact-corrected video microscopy measurements reveal an anomalous long-ranged like-charge attraction in the interparticle pair potential when the spheres are confined to a layer by even a single-charged glass surface. These attractions can be masked by electrostatic repulsions at low ionic strengths. Coating the bounding surfaces with a conducting gold layer suppresses the attraction. These observations suggest a possible mechanism for the anomalous confinement-induced attractions.
\end{abstract}

DOI: 10.1103/PhysRevE.76.041406

PACS number(s): 82.70.Dd, 05.40.-a, 61.20.-p

\section{INTRODUCTION}

The electrostatic interaction between charge-stabilized colloidal particles is mediated and modified by simple ions dissolved in a supporting electrolyte. For like-charged particles, the resulting effective interaction, described by the Poisson-Boltzmann mean-field theory [1], is predicted to be always purely repulsive [2-5] independent of the strength of the electrostatic coupling, the valency of the suspended ions, or the state of confinement of the suspension.

This reasonable prediction is known to fail under some conditions. For example, both simulations and non-meanfield theoretical studies predict the possibility of an attraction in bulk suspensions of like-charged colloidal particles if multivalent counterions are present (see [6,7] and references therein). This attraction is mediated by strong ion-ion correlations induced by coupling to the charged colloidal particles. Experimental observations have confirmed similar predictions for highly charged parallel plates [8] and cylinders [9].

These departures from mean-field behavior appear even in the so-called primitive model (PM), which treats both the colloidal particles and the dissolved ions as charged hard spheres and describes the suspending fluid as a uniform dielectric continuum. Similar effects are also predicted in more general models, when image charges [10], salt-specific dispersion forces [11], or the sizes of simple ions [12] become important.

In all such cases, mean-field theory fails because the relevant electrostatic interactions exceed the thermal energy scale over length scales of interest. Colloidal spheres in symmetric monovalent electrolytes, by contrast, are expected to satisfy the conditions of the mean-field approximation. Indeed, PM simulations of charged colloidal spheres in symmetric monovalent electrolytes are found to agree well with mean-field predictions [13-15], a class of systems for which Poisson-Boltzmann theory is expected to be accurate. Experimental results, however, have been more mixed. Direct measurements of colloidal pair interactions [16-18] in bulk dispersions qualitatively agree with mean-field predictions. When applied to dispersions confined to thin layers by charged glass surfaces, however, these same methods have repeatedly revealed long-ranged attractions [19-22] that are too strong to be accounted for by van der Waals interactions $[22,23]$ and are inconsistent with Poisson-Boltzmann theory [2-4]. Measurements on colloidal spheres confined by just a single glass wall, by contrast, have not revealed anomalous attractions [18,24-26]. These measurements were performed at much lower ionic strengths than those in thin samples, however. One explanation for this seeming inconsistency is that the appearance of confinement-induced like-charge attractions might depend strongly on the ionic concentration. Perhaps, then, even a single bounding surface could induce anomalous attractions if the ionic strength were in the appropriate range.

Another possible explanation is that the measurements reporting anomalous attractions among like-charged colloidal spheres were in error. Several experimental artifacts have been proposed that might mimic the reported observations. These include a geometric bias introduced by projecting a colloidal sphere's three-dimensional position onto the twodimensional focal plane [27], a statistical bias resulting from the experiments' limited sample size [24], nonequilibrium hydrodynamic interactions induced by small in-plane drifts [28], and uncorrected many-body contributions to the apparent pair potential [25]. Thermodynamic self-consistency checks demonstrate that none of these can account for the observed like-charge attractions in more recent experiments $[21,29,30]$.

Recently, a subtle imaging artifact in widely used particletracking algorithms [17] has been demonstrated to mimic long-ranged attractions in systems with purely repulsive pair interactions [31,32]. Its influence on the long-ranged repulsive pair potentials observed in low ionic strength bulk and surface experiments should be minimal, but the effect could play a major role at the smaller separations relevant for observations of like charge attractions [32]. Could the appearance of confinement-induced like charge be due entirely to experimental artifacts?

The experiments and simulations reported in this article confirm the appearance of confinement-induced like-charge attractions, even when all known experimental artifacts are taken into account. They furthermore contribute three insights into the effect's phenomenology: (i) Confinement by a single charged glass surface can induce anomalous attractions among charge-stabilized colloidal silica spheres. (ii) Confinement-induced attractions may be masked by electro- 


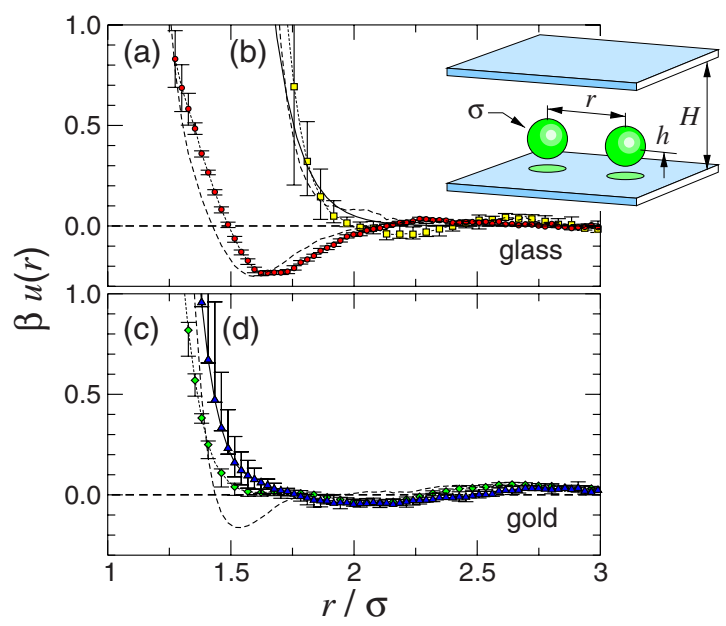

FIG. 1. (Color online) Measured pair potentials $u(r)$ for $\sigma$ $=1.58 \mu \mathrm{m}$ diameter silica spheres near a single wall $(H$ $\simeq 200 \mu \mathrm{m})$. Dashed curves are uncorrected results $\widetilde{u}(r)$. Discrete points have been corrected for imaging artifacts. Inset: schematic of the geometry. (a) Glass surface with $\kappa^{-1}=60 \pm 10 \mathrm{~nm}$ (circles) induces a long-ranged attraction. (b) Increasing the screening length to $\kappa^{-1} \approx 180 \mathrm{~nm}$ (squares) yields monotonic repulsion. The solid curve is a fit to Eq. (10) with $Z=6500 \pm 1000$. (c) Coating the confining surface with gold eliminates attractions at $\kappa^{-1} \approx 100 \mathrm{~nm}$ : Single surface (diamonds). (d) Parallel gold-coated surfaces separated by $H=15 \mu \mathrm{m}$ (triangles).

static repulsion at very low ionic strengths. (iii) Coating the confining surfaces with conducting gold layers eliminates the attraction, even under conditions of ionic strength that foster attractions in glass-bounded samples. These results are summarized in the data plotted in Fig. 1.

Section II describes the experimental and analytical methods used to obtain these results. Of particular importance are the methods introduced in Secs. II B and II C to measure and correct for imaging artifacts. The additional insights these experimental results provide into the nature of like-charge colloidal attractions suggest possible mechanisms for the effect. On this basis, we present an idealized model for confinement-induced like-charge attractions in Sec. III. This interpretation of our experimental results relies on the accuracy and efficacy of our analytical techniques, which we demonstrate through Monte Carlo simulations in Sec. IV. Section V summarizes our results and conclusions while placing them in the context of recent advances in the theory of macro-ionic interactions.

\section{ARTIFACT-FREE EQUILIBRIUM INTERACTIONS MEASUREMENTS}

\section{A. Experimental setup}

Our samples consist of uniform silica spheres $\sigma$ $=1.58 \pm 0.06 \mu \mathrm{m}$ in diameter (Duke Scientific Lot 24169) dispersed in deionized water and loaded into hermetically sealed sample volumes formed by bonding the edges of glass No. 1.5 coverslips to the surfaces of glass microscope slides separated by $H=200 \mu \mathrm{m}$ [33]. All glass surfaces were cleaned in a 4:1 mixture of sulfuric acid and hydrogen peroxide and rinsed in deionized water before assembly to increase their surface charge density. Access to the sample volume is provided by glass tubes bonded to holes drilled through the slides. Filling the tubes with mixed bed ion exchange resin maintains the ionic strength below $5 \mu \mathrm{M}$, corresponding to a Debye-Hückel screening length of $\kappa^{-1}$ $\approx 180 \mathrm{~nm}$. Removing the ion exchange resin and allowing the dispersions to equilibrate with air reduces the screening length to $\kappa^{-1} \approx 60 \mathrm{~nm}$.

We also prepared sample volumes with conducting inner surfaces by evaporating $10-\mathrm{nm}$ gold films with $10-\mathrm{nm}$ titanium wetting layers onto the glass surfaces before assembly. The metallic films have a resistivity of $50 \Omega / \square$ and are optically transparent. The electrodes were left floating. Studies of similar systems suggest a surface charge density of $-0.4 \mathrm{mC} / \mathrm{m}^{2}$ for the clean glass surfaces [34] and at least 100 times smaller for the gold films [35].

Samples were mounted on the stage of a Zeiss Axiovert 100 STV microscope and allowed to equilibrate at room temperatures $(T=297 \pm 1 \mathrm{~K})$. The silica spheres sediment into a dilute layer with their centers about $0.9 \mu \mathrm{m}$ above the lower wall. Thermally driven out-of-plane fluctuations are estimated to be smaller than $300 \mathrm{~nm}[21,24]$. In all the experiments, the colloidal areal density is $n \sigma^{2} \simeq 0.08$. The brightfield imaging system provides a magnification of $212 \mathrm{~nm} /$ pixel on a Hitachi TI-11A monochrome chargecoupled-device (CCD) camera. The resulting video stream was analyzed with standard methods of digital video microscopy [17] to measure the particles' positions, which then were used to estimate their effective pair potential.

\section{B. Measuring measurement errors}

Unlike artifacts arising from actual properties of the physical system, imaging artifacts result from misidentification of the spheres' centroids. At first blush, the bright-field image of a colloidal sphere appears as a bright region on a darker background. The centroid then may be identified with subpixel resolution as the brightness-weighted center of brightness [17]. In fact, a sphere's image is a projection of its far-field Mie scattering pattern [36], consisting of alternating dark and bright rings encircling the central intensity maximum. This more complicated pattern may be analyzed in a conventional manner [17] provided one sphere's image does not overlap with those of its neighbors. Distortions arising from overlapping scattering patterns lead to systematic deviations in the particles' apparent positions [31]. These distortions, in turn, distort estimates for the pair potential derived from the measured particles positions [31]. Because the errors are in the particle locations themselves, the resulting distortion of the pair potential is not detected by methods based on thermodynamic self-consistency [21,29,30].

Fortunately, distortions due to imaging artifacts can be corrected if the artifact's dependence on interparticle separation is known. Figure 2 shows two complementary methods for measuring this, one of which can be applied a posteriori to archival data without requiring additional calibration measurements. 
We explicitly measure overlap distortions by using holographic optical tweezers $[37,38]$ to hold two spheres at specified separations while a third is held far enough away to use as an undistorted reference. For each separation, we measure the apparent distance $\widetilde{r}$ between the closely spaced pair [Fig. 2(a)] and then independently measure their separations $r_{1}$ and $r_{2}$ from the reference sphere with the other sphere absent [Figs. 2(b) and 2(c)]. The first measure is skewed by the artifact. The two reference measurements are not. Consequently, their difference $r=r_{2}-r_{1}$ is an unbiased measurement of the real separation. The difference $\Delta(r)=\tilde{r}-r$ is a measurement of the artifact, whose separation dependence is plotted as circles in Fig. 2. As previously reported [31], these systematic deviations exceed the instrumental error bound for single-particle tracking [17] at separations relevant for interaction measurements. The data in Fig. 2 were obtained with a $100 \times$ NA 1.4 oil immersion objective, yielding an effective magnification of $135 \mathrm{~nm} /$ pixel. Comparable results can be obtained with the $60 \times$ objective used for interaction measurements and with one or two metal-coated surfaces.

This approach is accurate, but somewhat painstaking, and requires samples with prohibitively low areal densities [24]. We therefore introduce an alternative way to measure $\Delta(r)$ that relies on information already contained in the imaging data used to estimate the pair potential.

Some spheres in a given image will be far enough from all of their neighbors that their images are unaffected by overlap distortions. The image of such a sphere can be clipped from the larger field of view, duplicated, and used to construct composite two-sphere images at known separations $r$. Examples of such composite images created from displaced copies of a single sphere's image are shown in Figs. 2(d)-2(f). The apparent separation $\widetilde{r}$ in each composite image is then measured to obtain the difference $\Delta(r)=\widetilde{r}-r$, whose separation dependence is plotted as squares in Fig. 2. This method is based on the assumption that overlap artifacts result from the linear superposition of neighboring spheres' images. Its quantitative agreement with results obtained by explicit measurement justifies this assumption. Consequently, we use composite images to measure and correct for $\Delta(r)$ in each of our measurements of colloidal interactions.

\section{Measuring the pair potential}

We estimate the effective pair potentials of our confined dispersions by compiling the particles' apparent positions $\widetilde{\mathbf{r}}_{j}(t)$ into the single-particle distribution function

$$
\rho(\widetilde{\mathbf{r}}, t)=\sum_{j=1}^{N(t)} \delta\left(\widetilde{\mathbf{r}}-\widetilde{\mathbf{r}}_{j}(t)\right),
$$

where $N(t)$ is the number of particles in the visible area $A$ at time $t$. Data obtained over a period of about an hour [24] then are combined into the radial distribution function

$$
\widetilde{g}(\widetilde{r})=\frac{1}{n^{2}}\left\langle\rho\left(\widetilde{\mathbf{r}}^{\prime}-\widetilde{\mathbf{r}}, t\right) \rho\left(\widetilde{\mathbf{r}}^{\prime}, t\right)\right\rangle,
$$

where $n=\langle N(t)\rangle / A$ is the mean areal density. The angle brackets denote averages over $\widetilde{\mathbf{r}}^{\prime}$, angles, and time. In prin-

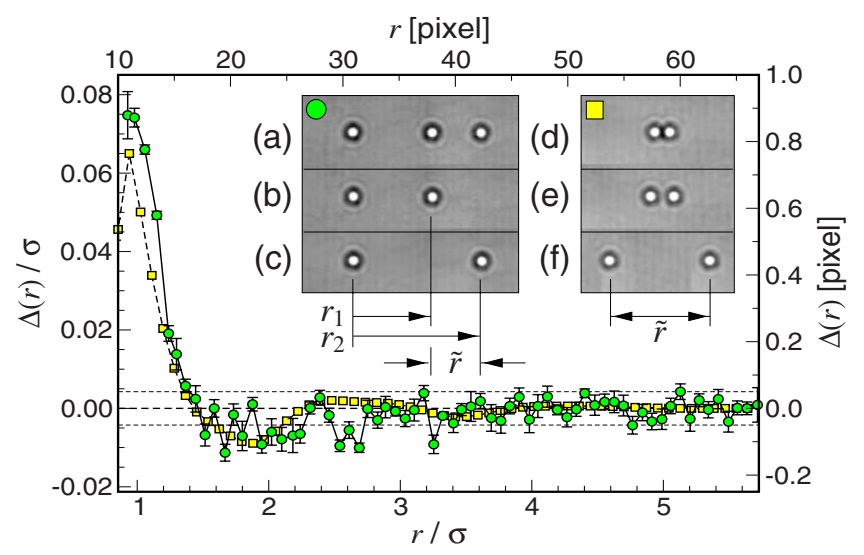

FIG. 2. (Color online) Measured apparent displacement $\Delta(r)$ from true separation $r$ due to an imaging artifact. Circles were obtained with holographic optical tweezer measurements (a), (b), and (c) and squares with composite images including (d), (e), and (f).

ciple, the pair correlation function can be inverted to obtain the particles' effective pair interaction potential. The imaging artifact, however, systematically distorts the pair separations used to compute $\widetilde{g}(\widetilde{r})$ and therefore distorts the estimated pair potential [31].

The undistorted pair correlation function $g(r)$ is related to $\tilde{g}(\widetilde{r})$ through conservation of probability,

$$
g(r) d r=\widetilde{g}(\widetilde{r}) d \widetilde{r} .
$$

Provided that the sample's areal density is low enough to preclude substantial three-body overlap distortions, this yields

$$
g(r)=\widetilde{g}(r+\Delta(r))\left[1+\frac{d}{d r} \Delta(r)\right] .
$$

Were the sample sufficiently dilute, the effective pair potential could be computed from $g(r)$ using the Boltzmann relation

$$
\beta u(r)=-\ln g(r),
$$

where $\beta^{-1}=k_{B} T$ is the thermal energy scale at temperature $T$. Comparatively weak many-body contributions to $g(r)$ can be corrected in either the hypernetted-chain (HNC) or PercusYevick (PY) approximations as [21,24,39]

$$
\beta u(r)=-\ln g(r)+ \begin{cases}n I(r) & (\mathrm{HNC}), \\ \ln [1+n I(r)] & (\mathrm{PY}),\end{cases}
$$

where the convolution integral

$$
I(r)=\int_{A}\left[g\left(r^{\prime}\right)-1-n I(r)\right]\left[g\left(\left|\mathbf{r}^{\prime}-\mathbf{r}\right|\right)-1\right] d^{2} r^{\prime}
$$

is solved iteratively, starting with $I(r)=0$. Differences between results obtained in the PY or HNC approximation provide estimates for the errors introduced by using approximate closure relations. At the comparatively low areal density of our experiments, the two closures agree with each 
other to within $1 \%$. Neither is large enough to affect the qualitative form of $u(r)$.

How imaging artifacts distort estimates for $u(r)$ depends on the relative positions and magnitudes of features in $\Delta(r)$ and $u(r)$. Applying Eq. (4) to Eq. (5) in the dilute limit yields

$$
\begin{aligned}
\beta \widetilde{u}(\widetilde{r}) & \approx \beta u(r-\Delta(r))+\frac{d \Delta(r)}{d r} \\
& \approx \beta u(r)-\beta \frac{d u(r)}{d r} \Delta(r)+\frac{d \Delta(r)}{d r} .
\end{aligned}
$$

This shows that even the sign of the error resulting from $\Delta(r)$ can depend on details of $u(r)$. Could the appearance of confinement-induced like-charge attractions be due entirely to experimental artifacts?

\section{Experimental results: Glass walls}

The a posteriori artifact corrections described in Secs. II B and II C were applied to the two data presented in Fig. 1. Pair potentials were tested for thermodynamic selfconsistency using previously reported methods $[29,30]$.

The data in Figs. 1(a) and 1(b) were obtained for colloidal silica spheres hovering above clean glass walls and demonstrate two of our principal points: Even a single glass wall can induce attractive interactions among like-charged colloidal spheres, and this attraction is not apparent at low ionic strengths.

The discrete points in Fig. 1 reflect estimates for the pair potential obtained with Eqs. (1)-(7), each of which was corrected for imaging artifacts with an individually measured calibration curve $\Delta(r)$. The dashed curves are results obtained without correcting for imaging artifacts. Under the conditions in Figs. 1(a) and 1(b), correcting for imaging artifacts leads to only small quantitative changes in the estimated pair potential, rather than qualitative changes as have been reported elsewhere [31]. More specifically, the minimum in the measured pair potential in Fig. 1(a) is not eliminated by correcting for imaging artifacts. A single clean glass wall, therefore, can induce like-charge attractions between nearby pairs of charge-stabilized spheres. All previous reports of like-charge attractions in equilibrium involved particles confined by pairs of parallel glass walls $[17,19,21,22,29]$.

This result should not be taken to contradict previous reports of colloidal interactions near single glass surfaces $[18,25,33,40-42]$, all of which described monotonically repulsive interactions. Unlike these previous reports, the data in Fig. 1(a) were obtained in deionized water that had been allowed to equilibrate with air, thereby increasing the concentration of dissolved monovalent ions to roughly $50 \mu \mathrm{M}$. Equilibrating the sample against mixed-bed ion exchange resin, as in previous reports, reduces the ionic strength, increases the range of the particles' electrostatic interaction, and yields the monotonically repulsive pair potential plotted in Fig. 1(b).

The purely repulsive pair potential may be compared with the screened-Coulomb form predicted by mean-field theory [1],

$$
\beta u(r)=Z^{* 2} \lambda_{B} \frac{e^{-\kappa r}}{r} .
$$

Here, $Z^{*}=Z \exp (-\kappa \sigma / 2) /(1+\kappa \sigma / 2)$ is the effective charge of a sphere of bare charge $Z$ in an electrolyte with DebyeHückel screening length $\kappa^{-1}$. This is related to the ionic concentration $n_{c}$ through $\kappa^{2}=8 \pi n_{c} \lambda_{B}$, where $\lambda_{B}=0.717 \mathrm{~nm}$ is the Bjerrum length in water at room temperature. Fitting Eq. (10) to the data in Fig. 1(b) yields $Z=6500 \pm 1000$, which agrees quantitatively with previous reports [24]. The fit screening length $\kappa^{-1}=180 \pm 10 \mathrm{~nm}$ corresponds to a concentration $n_{c}=2.8 \mu \mathrm{M}$ of monovalent ions. The result is plotted as a solid curve in Fig. 1(b).

The screening length obtained from Fig. 1(b) also is comparable to values obtained from previous measurements of colloidal interactions near single walls $[18,24-26]$. The attraction's dependence on ionic strength suggested by Figs. 1 (a) and 1(b) recalls a similar trend from the earliest report of attractions induced by two walls [22]. It seems plausible to suggest, therefore, that the attraction evident from the minimum in Fig. 1(a) either is eliminated by reducing the ionic concentration or else becomes masked by the stronger and longer-ranged screened-Coulomb repulsion. This also would explain why simulations of colloidal dispersions under salt-free conditions have found no evidence of confinement-induced attractions.

\section{E. Experimental results: Gold walls}

The data in Fig. 1(c) and 1(d) demonstrate our third principal observation: whereas a single glass surface can induce like-charge colloidal attractions, even two gold surfaces do not. These measurements were performed under comparable conditions of ionic strength to that in Fig. 1(a). Consequently, the range of the observed repulsion is comparable in both cases. The data in Fig. 1(c) were obtained for particles sedimented onto a single conducting surface in a $200-\mu \mathrm{m}$-thick sample chamber. Reducing the separation between gold-coated surfaces might be expected to strengthen any confinement-induced attraction because similar trends have been reported in previous studies on clean glass surfaces $[17,21]$. In fact, as shown in Fig. 1(d), reducing the interwall separation to $H=15 \mu \mathrm{m}$ while maintaining the ionic strength around $10 \mu \mathrm{M}$ has no significant influence on the measured pair potential.

The data in Fig. 1(c) demonstrate the importance of accounting for the imaging artifact. The uncorrected data, plotted as a dashed curve, display a marked minimum at a center-to-center separation of $1.5 \sigma$. This minimum is entirely eliminated by measuring $\Delta(r)$ for this data set and using it to correct $\widetilde{g}(\widetilde{r})$ according to Eq. (4). We therefore agree with the authors of Ref. [31] that imaging artifacts can create the appearance of attractive interactions where none exist. The data in Fig. 1(a) demonstrate, on the other hand, that this is not necessarily the case.

\section{INTERPRETATION}

It is possible that the gold surfaces' conductivity alone could suppress like-charge attractions-for instance, through 
a mechanism based on image charges. This would not explain, however, why glass walls induce attractions in the first place. Non-mean-field treatments of macroionic interactions [43-47] suggest that charged surfaces can induce oscillatory correlations in the distribution of simple ions surrounding charged colloidal spheres. Such local departures from electroneutrality could inject extra counterions between pairs of spheres, thereby inducing an effective attraction.

Modeling the predicted diffuse space charge density as a discrete point charge $q$, midway between the spheres' centers, yields the phenomenological pair potential [26]

$$
\beta u(r)=Z^{*} \lambda_{B}\left(Z^{*} \frac{e^{-\kappa r}}{r}-4 q \frac{e^{-\kappa r / 2}}{r}\right) .
$$

Equation (11) agrees well with the data in Fig. 1(a) for $Z$ $=6500 \pm 1000, \kappa^{-1}=60 \pm 10 \mathrm{~nm}$, and $q=10 \pm 5$. It also agrees with the purely repulsive potential in Fig. 1(b) for $Z$ $=7000 \pm 1000, \kappa^{-1}=198 \pm 10 \mathrm{~nm}$, and $q=7 \pm 3$. In this interpretation of the data, the wall-induced attraction is indeed masked by the electrostatic repulsion at low enough salt concentrations.

Whereas Fig. 1(a) suggests that the wall's surface charge injects an equivalent of ten counter-ions between the spheres and Fig. 1(b) is consistent with this interpretation, the data in Figs. 1(c) and 1(d) both are consistent with $q=0$. The other fitting parameters are $Z=8000 \pm 1000$ and $\kappa^{-1}=95 \pm 10 \mathrm{~nm}$ for Fig. 1(c) and $Z=7000 \pm 1000$ and $\kappa^{-1}=105 \pm 10 \mathrm{~nm}$ for Fig. 1(d). One interpretation of this result is that the weakly charged gold walls induce no attraction because they have no counter-ions to contribute. Even a single glass wall, by contrast, carries enough charge to induce nonmonotonic correlations in the distribution of simple ions and thus to qualitatively alter the dynamics of nearby charged colloidal spheres.

Recent results on colloidal polystyrene spheres confined to the air-water interface suggest another possible explanation for our observations [48]. These interfacial colloids also display strong long-ranged attractive interactions that contrast with predictions [49]. This was interpreted as resulting from a nonuniform distribution of charged groups on the particles' surfaces. Fluctuations in neighboring particles' dipole moments then could induce long-ranged attractions. The proposed connection between nonuniform surface charge and the measured attraction has proved controversial [50,51]. Furthermore, the charged groups in our silica particles result from dissociation of silanol groups, which are uniformly distributed on the spheres' surfaces. It seems unlikely, therefore, that dipolar interactions due to surface charge inhomogeneities are responsible for the confinement-induced like-charge attractions that we observe.

\section{SIMULATIONS}

The results reported in Sec. II rely on the methods described in Secs. II B and II C for measuring and correcting imaging artifacts. Equation (4), in particular, does not account for three- and higher-body overlap distortions and so might yield erroneous results if particles tend to form clusters. To assess our method's accuracy and robustness, we performed Monte Carlo simulations of two-dimensional col-

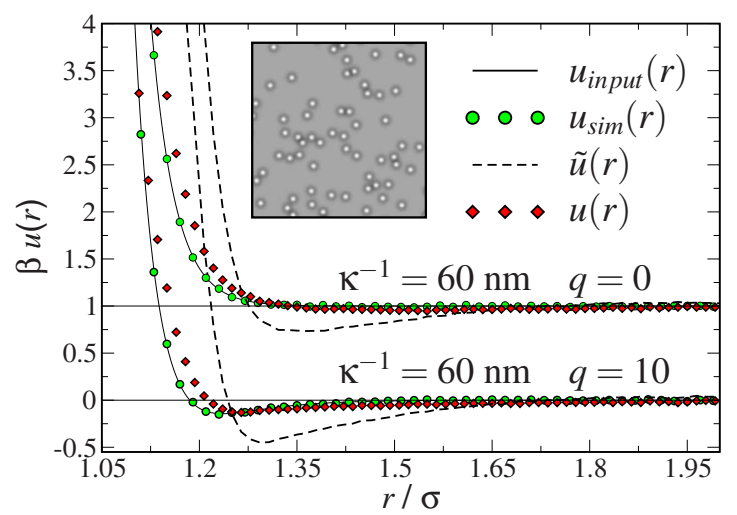

FIG. 3. (Color online) Monte Carlo tests of colloidal interaction measurements based on particle imaging. Hard disks interacting with specified potentials $u_{\text {input }}(r)$ (solid curves) yielded particle distributions that were inverted to recover the pair potential $u_{\text {sim }}(r)$ (circles). Convolving the distributions with a single-particle image yields synthetic multiparticle images (inset) that were analyzed to obtain artifact-distorted pair potentials $\widetilde{u}(r)$ (dashed curves). Correcting the artifact with Eq. (4) yields $u(r)$ (diamonds), which agrees with the input potential.

loidal dispersions at areal densities $n \sigma^{2}=0.08$, interacting through phenomenological potentials describing the experimental systems in Fig. 1(a) and 1(c). In each simulation, $N$ $=4600$ particles of diameter $\sigma=1.5 \mu \mathrm{m}$ were allowed to diffuse in a two-dimensional box of size $L=240 \sigma$ with periodic boundary conditions for a total of 11000 Monte Carlo sweeps. To avoid boundary effects, only particles in a smaller region of size $\ell=80 \sigma$ were analyzed. In both simulations, the particles were given an effective charge $Z$ $=6500$ and interacted through the potential in Eq. (11).

The solid curves in Fig. 3 show the potentials $u_{\text {input }}(r)$ used as inputs to simulations at $\kappa^{-1}=60 \mathrm{~nm}$ and $q=0$ or $q$ $=10$. Using the simulated particle positions as inputs to Eqs. (1), (2), (6), and (7) yields values for the recovered potential $u_{\text {sim }}(r)$, which are plotted as circles in Fig. 3. Statistical errors are smaller than $0.01 k_{B} T$ and so are smaller than the plot symbols. Agreement between $u_{\text {sim }}(r)$ and $u_{\text {input }}(r)$ confirms the correct implementation of our simulation and of the liquid structure analysis because the simulated particle positions are not displaced by imaging distortions.

To simulate the effects of imaging distortions, we convolve $\rho(\mathbf{r}, t)$ with an idealized single-sphere image to create synthetic images such as the example in the inset to Fig. 3. These are then analyzed with the same methods [17] used for experimental data to obtain distorted particle distributions $\rho(\widetilde{\mathbf{r}}, t)$ from which we compute the distorted apparent pair potential $\widetilde{u}(r)$. In both cases, the simulated imaging artifact introduces spurious long-ranged attractions into $\widetilde{u}(r)$, which are plotted as dashed curves in Fig. 3.

Finally, we processed the measured coordinates according to Eqs. (2), (4), and (6) to extract the artifact-corrected pair potentials $u(r)$. The correction curve $\Delta(r)$ was computed from the single-sphere image, so that the simulated results would most closely resemble the experimental data presented in Sec. II. The results, plotted as diamonds in Fig. 3, differ 
only slightly from the input potential $u_{\text {input }}(r)$, with discrepancies suggesting the limited influence of many-particle clusters at the experimental areal density.

The a posteriori procedure introduced in Sec. II C for correcting imaging artifacts correctly eliminated the spurious minimum in the data with $q=0$ and only slightly diminished the genuine minimum in the data at $q=10$. These results confirm our procedure's efficacy for correcting imaging artifacts in measurements of colloidal interactions, at least under the experimental conditions of the present study.

\section{CONCLUSION}

We have demonstrated that charged glass surfaces induce anomalous attractions between nearby charge-stabilized spheres, even when account is taken of all known experimental artifacts. Such attractions can be masked by longranged electrostatic repulsions at the lowest ionic strengths. This poses the additional challenge for simulations that anomalous attractions may not be apparent in systems without added salt. Replacing charged glass surfaces with con- ducting metal surfaces eliminates the anomalous attractions. This suggests a primary role for the surface charge in mediating confinement-induced like-charge attractions.

We speculate that charged surfaces induce like-charge attractions by inducing nonmonotonic correlations in the distribution of simple ions surrounding the macroions. In a crude sense, particles are attracted to counter-ions that the surface forces between them. Theories for macro-ionic interactions incorporating such correlations indeed predict likecharge attractions in confined [43,44] and crowded [45-47,52] dispersions, but are controversial [5,53-55]. Recent large-scale simulations $[7,56-58]$ strengthen the case for correlation-induced like-charge attractions, having revealed high-order correlations in the distribution of simples ions that are not captured by the mean-field approximation. These simulations show the onset of many-body cohesion that may be related to the wall-induced pair attraction that we observe.

\section{ACKNOWLEDGMENT}

This work was supported by the donors of the Petroleum Research Fund of the American Chemical Society.
[1] W. B. Russel, D. A. Saville, and W. R. Schowalter, Colloidal Dispersions (Cambridge University Press, Cambridge, England, 1989).

[2] J. C. Neu, Phys. Rev. Lett. 82, 1072 (1999).

[3] J. E. Sader and D. Y. C. Chan, J. Colloid Interface Sci. 213, 268 (1999).

[4] J. E. Sader and D. Y. C. Chan, Langmuir 16, 324 (2000).

[5] E. Trizac and J.-L. Raimbault, Phys. Rev. E 60, 6530 (1999).

[6] L. Belloni, J. Phys.: Condens. Matter 12, R549 (2000).

[7] P. Linse, Adv. Polym. Sci. 185, 111 (2005).

[8] P. Kékicheff, S. M. Celja, T. J. Senden, and V. E. Shubin, J. Chem. Phys. 8, 6098 (1999).

[9] J. L. Sikorav, J. Pelta, and F. Livolant, Biophys. J. 67, 1387 (1994).

[10] R. Kjellander and S. Marcelja, Chem. Phys. Lett. 112, 49 (1984).

[11] F. W. Tavares, D. Bratko, H. W. Blanch, and J. M. Prausnitz, J. Phys. Chem. B 108, 9228 (2004).

[12] H. Greberg and R. Kjellander, J. Chem. Phys. 108, 2940 (1998)

[13] P. Linse, J. Phys.: Condens. Matter 14, 13449 (2002).

[14] R. Messina, C. Holm, and K. Kremer, Phys. Rev. Lett. 85, 872 (2000).

[15] J. Wu, D. Bratko, and J. M. Prausnitz, Proc. Natl. Acad. Sci. U.S.A. 95, 15169 (1998).

[16] J. C. Crocker and D. G. Grier, Phys. Rev. Lett. 73, 352 (1994).

[17] J. C. Crocker and D. G. Grier, J. Colloid Interface Sci. 179, 298 (1996).

[18] K. Vondermassen, J. Bongers, A. Mueller, and H. Versmold, Langmuir 10, 1351 (1994).

[19] M. D. Carbajal-Tinoco, F. Castro-Román, and J. L. ArauzLara, Phys. Rev. E 53, 3745 (1996).

[20] J. C. Crocker and D. G. Grier, Phys. Rev. Lett. 77, 1897
(1996).

[21] Y. Han and D. G. Grier, Nature (London) 424, 267 (2003).

[22] G. M. Kepler and S. Fraden, Phys. Rev. Lett. 73, 356 (1994).

[23] B. A. Pailthorpe and W. B. Russel, J. Colloid Interface Sci. 89, 563 (1982).

[24] S. H. Behrens and D. G. Grier, Phys. Rev. E 64, 050401(R) (2001).

[25] M. Brunner, C. Bechinger, W. Strepp, V. Lobaskin, and H. H. von Grunberg, Europhys. Lett. 58, 926 (2002).

[26] D. G. Grier and Y. Han, J. Phys.: Condens. Matter 16, S4145 (2004).

[27] K. S. Rao and R. Rajagopalan, Phys. Rev. E 57, 3227 (1998).

[28] Y. O. Popov, J. Colloid Interface Sci. 252, 320 (2002).

[29] Y. Han and D. G. Grier, Phys. Rev. Lett. 92, 148301 (2004).

[30] Y. Han and D. G. Grier, J. Chem. Phys. 122, 164701 (2005).

[31] J. Baumgartl and C. Bechinger, Europhys. Lett. 71, 487 (2005).

[32] J. Baumgartl, J. L. Arauz-Lara, and C. Bechinger, Soft Mater. 2, 631 (2006).

[33] S. H. Behrens and D. G. Grier, Phys. Rev. E 64, 050401(R) (2001).

[34] S. H. Behrens and D. G. Grier, J. Chem. Phys. 115, 6716 (2001).

[35] M. A. Henderson, Surf. Sci. Rep. 46, 5 (2002).

[36] C. F. Bohren and D. R. Huffman, Absorption and Scattering of Light by Small Particles (Wiley Interscience, New York, 1983).

[37] E. R. Dufresne and D. G. Grier, Rev. Sci. Instrum. 69, 1974 (1998).

[38] M. Polin, K. Ladavac, S.-H. Lee, Y. Roichman, and D. G. Grier, Opt. Express 13, 5831 (2005).

[39] E. M. Chan, J. Phys. C 10, 3477 (1977).

[40] J. Bongers, H. Manteufel, H. Versmold, and K. Vondermaßen, 
J. Chem. Phys. 108, 9937 (1998).

[41] R. V. Durand and C. Franck, Phys. Rev. E 61, 6922 (2000).

[42] C. Franck, M. Covelli, and R. V. Durand, Phys. Rev. E 67, 041402 (2003).

[43] D. Goulding and J.-P. Hansen, Mol. Phys. 95, 649 (1998).

[44] D. Goulding and J. P. Hansen, Europhys. Lett. 46, 407 (1999).

[45] R. Hastings, J. Chem. Phys. 68, 675 (1978).

[46] B. P. Lee and M. E. Fisher, Europhys. Lett. 39, 611 (1997).

[47] M. D. Carbajal-Tinoco and P. Gonzalez-Mozuelos, J. Chem. Phys. 117, 2344 (2002).

[48] W. Chen, S. Tan, Z. Huang, T.-K. Ng, W. T. Ford, and P. Tong, Phys. Rev. E 74, 021406 (2006).

[49] A. J. Hurd, J. Phys. A 18, L1055 (1985).
[50] A. Dominguéz, D. Frydel, and M. Oettel, e-print arXiv:0706.3977

[51] T.-K. Ng and Y. Zhou, e-print arXiv:0708.2518.

[52] A. R. Denton, Phys. Rev. E 70, 031404 (2004).

[53] E. Trizac, Phys. Rev. E 62, R1465 (2000).

[54] E. M. Mateescu, Phys. Rev. E 64, 013401 (2001).

[55] E. Trizac and J. L. Raimbault, Phys. Rev. E 64, 043401 (2001).

[56] E. Allahyarov, I. D’Amico, and H. Löwen, Phys. Rev. E 60, 3199 (1999).

[57] A. Delville, J. Phys. Chem. B 109, 8164 (2005).

[58] P. B. Warren, Phys. Rev. E 73, 011411 (2006). 